\title{
SCALABLE LIQUID METAL THIN LINE PATTERNING FOR PASSIVE ELECTRONIC COMPONENTS USING SOFT LITHOGRAPHY
}

\author{
M. Kim*, H. Alrowais, S. Pavlidis and O. Brand
}

School of Electrical and Computer Engineering, Georgia Institute of Technology, Atlanta, GA, USA

Institute for Electronics and Nanotechnology, Georgia Institute of Technology, Atlanta, GA, USA

\section{ABSTRACT}

This paper presents a size-scalable and residue-free liquid metal patterning technique for all-soft passive electronic components using soft lithography and gallium-based liquid metal (EGaIn, Eutectic Gallium-Indium Alloy). Using the proposed technique, uniform and smooth EGaIn lines with widths ranging from single micrometers to several millimeters are patterned on a PDMS mold at room temperature in ambient pressure. To highlight the process capabilities, the electrical characteristics of fabricated passive components, such as resistors, interdigitated capacitors, as well as vertical interconnects using flexible-through-PDMS vias (fTPVs) are investigated under bending and twisting deformation.

\section{INTRODUCTION}

Light-weight, flexible and stretchable electronics have gained significant attention with possible epidermal electronic applications for human health and performance monitoring [1]. One approach to fabricate stretchable electronics is to combine soft substrate materials with conventional metal conductors using wave-like patterns to improve stretchability [1-2].

An alternative approach is to use stretchable conductor materials, such as conducting liquids, to open the path for all-soft, reconfigurable and self-healing electronics [3-4]. Among available liquid conductors, EGaIn is of particular interest due to its excellent electrical and mechanical properties, including high electrical conductivity $\left(3.4 \times 10^{6} \mathrm{~S} / \mathrm{m}\right)$, low melting temperature $\left(15^{\circ} \mathrm{C}\right)$, and low toxicity [4-5]. However, scalable EGaIn patterning ranging from the $\mu \mathrm{m}-\mathrm{mm}$ range remains a challenge [4], especially with commonly used techniques such as microfluidic injection, stencil or inkjet printing. Microtransfer molding has recently been demonstrated as promising technology to fabricate micron-scale EGaIn lines [5].

This paper advances microtransfer molding of liquid metals by demonstrating size-scalable, uniform and residue-free EGaIn patterning, and its application to all-soft passive electronic components and circuits (Figure 1).

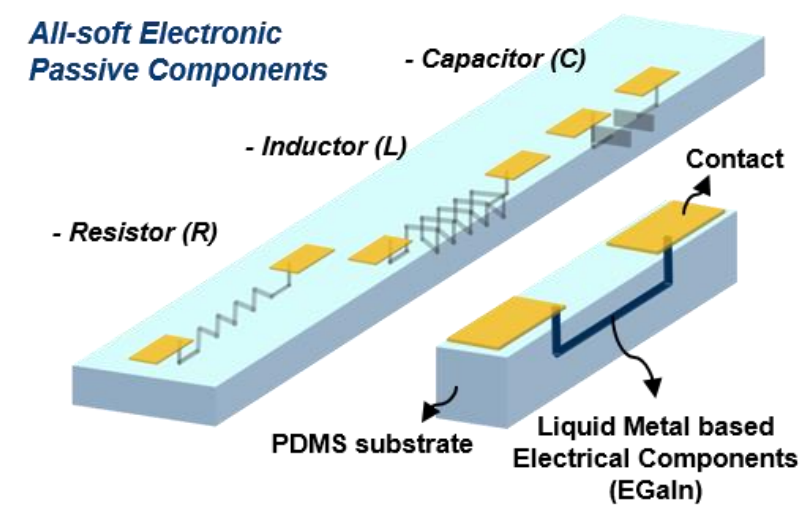

Figure 1: Concept of all-soft (flexible and stretchable) passive electronic components using gallium-based liquid metal (Eutectic Gallium-Indium Alloy, EGaIn) and PDMS as soft materials.
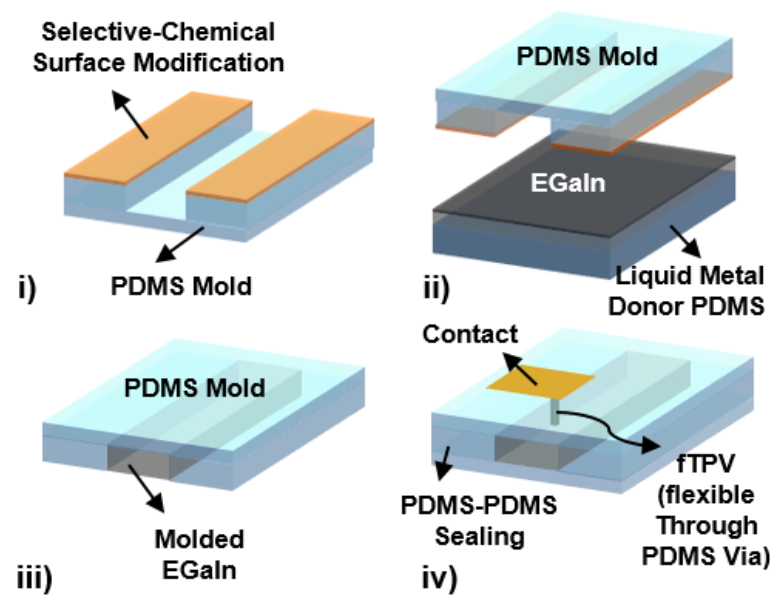

Figure 2: Scalable thin liquid metal line fabrication process: i) chemical surface modification of PDMS mold, ii)-iii) microtransfer molding of gallium-based liquid metal into PDMS mold, and iv) PDMS cover with contact and flexible through-PDMS via.
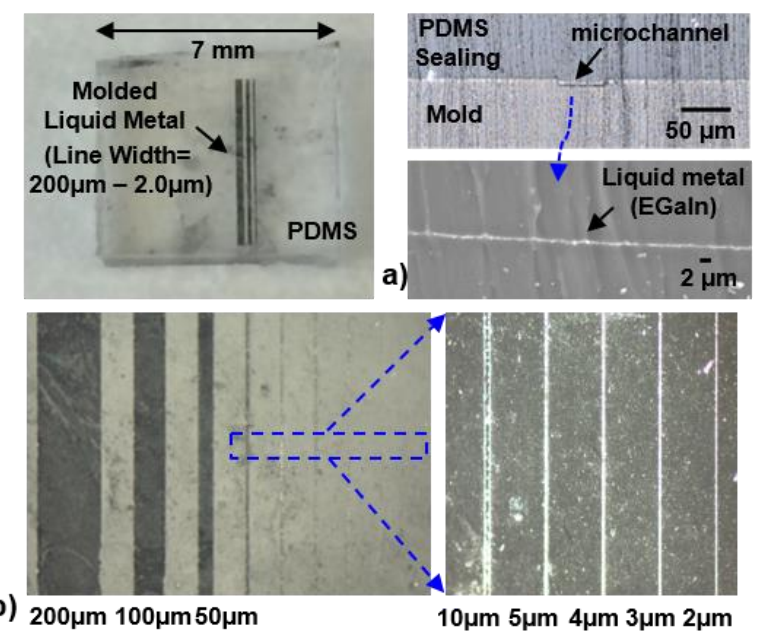

Figure 3: Scalable and uniform liquid metal lines patterned using microtransfer molding: (a) cross-sectional view of the EGaIn molded microchannel with $50 \mu \mathrm{m}$ width (top) and enlarged view of the molded EGaIn line with $300 \mathrm{~nm}$ thickness (bottom), and (b) molded liquid metal lines with widths from $2 \mu \mathrm{m}$ to $200 \mu \mathrm{m}$.

\section{DESIGN AND FABRICATION}

Figure 2 shows the EGaIn patterning process, which utilizes a PDMS mold with embedded microchannels. The surface of the PDMS mold is first selectively chemically modified using toluene for selective wetting. Then, the PDMS mold is pressed onto a donor substrate coated with the liquid metal film, and separated from it. Liquid metal residue on the PDMS mold outside of the channels can now be effectively transferred to a sacrificial PDMS layer. Finally, the PDMS mold with liquid-metal-filled channels is covered with an additional PDMS layer, which has embedded liquid-metal-filled through-PDMS vias for vertical interconnection.
Solid-State Sensors, Actuators and Microsystems Workshop Hilton Head Island, South Carolina, June 5-9, 2016 
Using this improved process sequence, EGaIn lines with widths ranging from $2 \mu \mathrm{m}$ to $200 \mu \mathrm{m}$ were patterned simultaneously, showing uniform and smooth EGaIn films inside of the PDMS (Figure 3(a)-(b)) and almost no residues between EGaIn features.

\section{RESULT AND DISCUSSION}

Figure 4(a) shows an embedded resistor, formed by a resistor line with $5 \mu \mathrm{m}$ width, connected with $50 \mu \mathrm{m}$ wide conductor lines. For high-density integration, vertical integration was demonstrated using fTPVs (aspect ratio=1.7-5.7, Figure 4(b)). Figure 4(c) and (d) compare the calculated and measured resistances of the resistors and fTPVs, respectively, with $<4 \%$ deviation between the calculated and measured values. Figure 5(a) and (b) show an all-soft interdigitated capacitor, having 30 interdigitated electrodes (IDEs), and the measured capacitance as a function of number of IDEs, which agrees well with the simulated values ( $<6 \%$ deviation).

To demonstrate bendability and stretchability, we investigated the electrical characteristics of the embedded resistor by applying bending and torsional forces (Figure 6(a)-(b)). While the resistance increases if the resistor is bend in length-direction, it decreases if bend in width-direction. For a minimal radius of $13.5 \mathrm{~mm}$, the measured relative resistance changes were $<13 \%$. Upon twisting, relative resistance changes were $<3 \%$, indicating that twisting forces have a minor effect because the resistor shape can be maintained.

Finally, a commercial LED was integrated on top of a soft circuit and subjected to bending and torsional forces (Figure 6(c)), demonstrating its electrical functionality under deformation.

\section{CONCLUSION}

A size-scalable EGaIn patterning and vertical integration technique is demonstrated to fabricate all-soft passive components and circuits for wearable electronics applications.

\section{ACKNOWLEDGEMENT}

This work was performed in part at the Georgia Tech Institute for Electronics and Nanotechnology, a member of the National Nanotechnology Coordinated Infrastructure, which is supported by the National Science Foundation (Grant ECCS-1542174), and travel support has been generously provided by the Transducer Research. a)
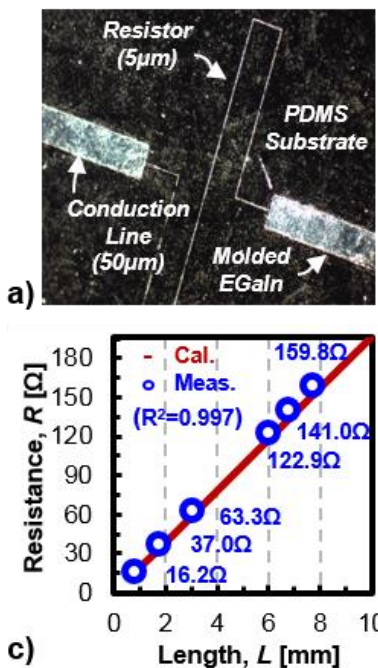
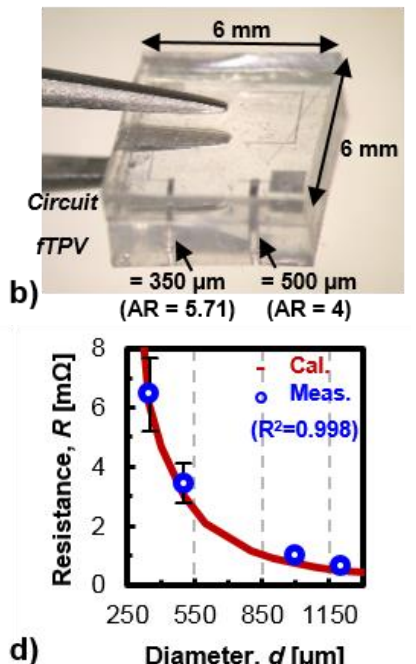

Figure 4: (a) Fabricated soft circuit with serpentine-shaped resistor $(5 \mu \mathrm{m})$ and conductor $(50 \mu \mathrm{m})$ lines; (b) vertical circuit integration using flexible through-PDMS vias (fTPVS); calculated and measured resistance of (c) conductor line as a function of line length, and (d) fTPV as a function of via diameter. a)
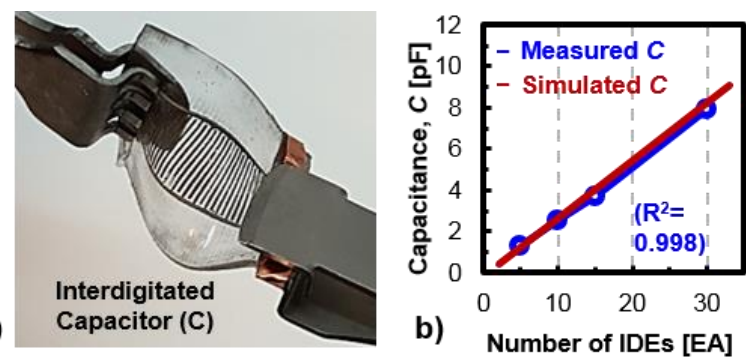

Figure 5: (a) Liquid-metal-embedded all-soft interdigitated capacitor and (b) simulated and measured capacitance as a function of number of interdigitated electrodes (IDEs).
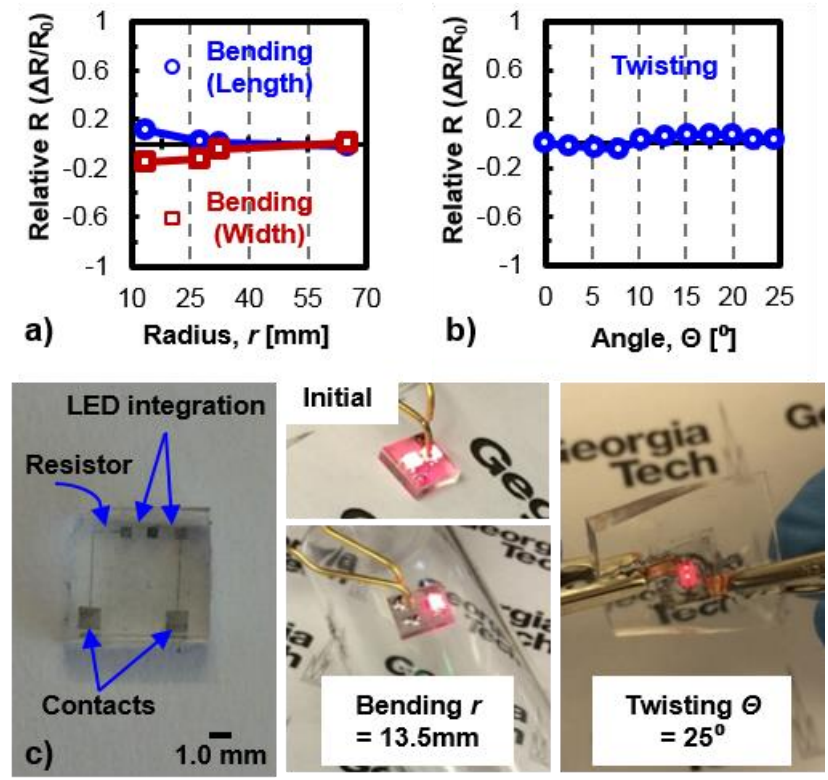

Figure 6: Resistance change of the embedded resistor soft-circuit to (a) bending and (b) torsional forces, and (c) system-level flexibility demonstration by applying bending and twisting forces to LED-integrated soft circuit.

\section{REFERENCES}

[1] D.-H. Kim, N. Lu, R. Ma, Y.-S. Kim, R.-H. Kim, S. Wang, J. Wu, S. M. Won, H. Tao, A. Islam, K. J. Yu, T.-i. Kim, R. Chowdhury, M. Ying, L. Xu, M. Li, H.-J. Chung, H. Keum, M. McCormick, P. Liu, Y.-W. Zhang, F. G. Omenetto, Y. Huang, T. Coleman, and J. A. Rogers, "Epidermal electronics", Science, 333, 838 (2011).

[2] D.-H. Kim, and J. A. Rogers, "Stretchable Electronics: materials strategies and devices", Advanced Materials, 20, 4887 (2008).

[3] M. D. Dickey, "Emerging application of liquid metals featuring surface oxides", ACS Applied Materials \& Interfaces, 6, 18369 (2014).

[4] I. D. Joshipura, H. R. Ayers, C. Majidi, and M. D. Dickey, "Method to pattern liquid metals", Journal of Materials Chemistry C, 3, 3834 (2015).

[5] B. A. Gozen, A. Tabatabai, O. B. Ozdoganlar, and C. Majidi, "High-density soft-matter electronics with micron-scale line width”, Advanced Materials, 26, 5211 (2014).

\section{CONTACT}

*M. Kim, tel: +1-404-317-4892; mgk@gatech.edu, and O. Brand, tel: +1-404-894-9425; oliver.brand@ece.gatech.edu. 